[7-10]. Research has been carried out by implementing quantitative ultrasound (QUS) analysis which is a rapid process to detect microstructural variation or heterogeneity in the margin of breast tumor [11]. In particular, low-frequency ultrasound is instrumental in detecting cancer and tissue characteristics. Low-frequency ultrasound is conventionally used at large scale tissues. But in the case of margin detection, the application depth is small enough to demonstrate high-frequency ultrasound for better resolution [11].

QUS is used to assess detailed tissue properties beyond the time domain into the frequency domain rather than obtaining direct images like medical ultrasonography [12-14]. Among various parameters of QUS, peak density of the frequency spectrum is one of the most responsive ones toward the structural variation [11]. Peak density is the number of peaks and valleys present in the frequency spectrum of the ultrasound signal. While sound travels in a heterogeneous medium and collides with different structures, it scatters in all directions. The scattered wave contains different pressure levels than the incident wave. The scattered wave is different from the transmitted or radiated wave because waves scattered from different microstructures interfere with each other [15]. The scattered wave cannot be expressed as a simple function of attenuation or reflection. While using a broadband transducer, the resultant scattered pressure amplitude for each frequency level generates a spectrum for the whole bandwidth. The total number of peaks and valleys of that spectrum can be presented as peak density. A change in the characteristics of the propagation medium translates into variation in peak density patterns [16, 17]. Doyle et al. showed that peak density is responsive toward benign and malignant tissue $[11,17,18]$. Stromer et al. worked with different signal processing parameters such as different types of windowing functions to get the best response in peak density. But in these studies, the thickness of the sample or tissue was neglected as a parameter for evaluating peak density $[19,20]$. Since the frequency is at megahertz level, the attenuation should be very sensitive toward the change in travel distance which could impact the peak density. Also, the heterogeneity will expand with increasing tissue thickness in that direction. For these reasons, tissue thickness should be considered as a controlling parameter of peak density measurement. Furthermore, since peak density is measured from the frequency spectrum of the scattered wave, it is important to understand the effect of sound scattering on peak density evaluation. To the author's understanding, no study has been conducted on explaining sound scattering to establish it as a function of peak density as well as evaluating the significance level of other parameters on peak density measurement.

This study focuses on developing a relationship between peak density and acoustic scattering, hence peak density in soft tissues containing hard scatterers and particles. To relate the effect of different geometrical and microstructural parameters on the scattering and peak density, peak density was measured in different scattering conditions in a pitchcatch method using tissue phantom containing different scatterer sizes and thicknesses. Furthermore, to evaluate spatial distribution impact on the resulting peak density, samples with different scatterer densities were tested. Analytical evaluation and computational calculations were conducted to theoretically relate these parameters. Analytically, the peak density was calculated from Faran's single scattering model using a spherical scatterer while varying its diameter [21]. Peak density was also measured from the computational model and experiment while varying the scatterer size, spatial distance (by changing the volume ratio of scatterers), and ultrasound travel distance (by varying the sample thickness). Agarose phantom with glass microspheres was used with 3 different levels of phantom thickness, microsphere size, and number. 25-MHz transducer was used to perform the ultrasound analysis in a pitch-catch method. The received scattered signal was transformed using Fast Fourier Transform (FFT) and passed through signal processing steps before the peak density was evaluated.

\section{Analytical study}

When sound as a plane wave travels and collides with a spherical structure, it scatters away at every direction [21, 22]. In this case, because the incident wave is considered as spherical, the incident sound pressure wave $\left(P_{i}\right)$ can be expressed as [21],

$P_{i}=P_{o} \sum_{l=0}^{\infty}(2 l+1) i j_{l}(\mathrm{kr}) P_{l}(\cos \theta)$

Here, $P_{o}$ is the amplitude of the incident wave pressure, $j_{l}$ is the spherical Bessel function of the second kind and $P_{l}$ is the Legendre polynomial, respectively, $k$ is the wavenumber, and $r$ and $\theta$ correspond to the polar coordinate.

In this model, the scatterer is considered elastic. Therefore, the shear and compressional waves are considered to measure the scattered wave pressure. So, the boundary conditions were (1) the fluid pressure incident $\left(P_{i}\right)$ and scattered $\left(P_{s}\right)$ pressure fields to be equal to normal stress component of solid, (2) normal displacements in the fluid and solid to be equal, and (3) the shear stress to be zero which are shown through the following equation:

$$
\begin{aligned}
& P_{i}+P_{s}=-T_{\mathrm{rr}} \\
& u_{i, r}+u_{s, r}=-u_{\text {solid }, r}
\end{aligned}
$$

\section{SN Applied Sciences}


$T_{r \theta}+T_{r \Phi}=0$

Here $T_{r r}, T_{r \theta}$, and $T_{r \Phi}$ are the stress tensor of the solid, respectively, and $u_{i, r}$ and $u_{s, r}$ normal displacement components (incident and scattered, respectively) of fluid, and $-u_{\text {solid, } r}$ is the normal displacement component of the solid in the spherical coordinates, respectively.

After sound scattering, the scattered pressure $\left(P_{s}\right)$ wave can be expressed as

$P_{s}=-P_{o} \sum_{l=0}^{\infty}(2 l+1)(-i)^{l+1} \sin \eta_{l} e^{i \eta_{l}} h_{l}^{(2)}(\mathrm{kr}) P_{l}(\cos \theta)$

Since the focus was on pitch-catch mode, at the far-field $(r \rightarrow \infty)$ sound pressure of the scattered field is described as

$P_{s, f a r}=-\frac{P_{0}}{k_{3} r} \sum_{l=0}^{\infty}(2 l+1) \sin \eta_{l} e^{i \eta_{l}} P_{l}(\cos \theta)$

Here, $\eta_{l}$ is the scattering phase angle of $/$ th wave due to scatterer properties; $h_{l}^{(2)}$ is the Hankel function. The phase shift angle was expressed as

$\eta_{l}=\tan ^{-1}\left[\frac{\tan \left(\delta_{l}\left(k_{3} a\right)\right)\left(\tan \Phi_{l}+\tan \alpha_{l}\left(k_{3} a\right)\right)}{\tan \Phi_{l}+\tan \left(\beta_{l}\left(k_{3} a\right)\right)}\right]$

where

$\tan \alpha_{l}\left(k_{3} a\right)=-\frac{\left(k_{3} a\right) j_{l}^{\prime}\left(k_{3} a\right)}{j_{l}\left(k_{3} a\right)}$

$\tan \beta_{l}\left(k_{3} a\right)=-\frac{n_{l}^{\prime}\left(k_{3} a\right)}{n_{l}\left(k_{3} a\right)}$

$\tan \delta_{l}\left(k_{3} a\right)=-\frac{j_{l}\left(k_{3} a\right)}{n_{l}\left(k_{3} a\right)}$

$\tan \Phi_{l}=-\frac{\rho_{3}}{\rho_{1}} \tan \xi(k a, v)$
Table 1 Physical parameters used for the analytical model

\begin{tabular}{ll}
\hline Parameter & Value \\
\hline Poisson's ratio $(v)$ & 0.22 \\
Compressional wave speed in scatterer $\left(c_{1}\right)$ & $5770 \mathrm{~m} / \mathrm{s}$ \\
Shear wave speed in scatterer $\left(c_{2}\right)$ & $3430 \mathrm{~m} / \mathrm{s}$ \\
Sound wave speed in fluid $\left(c_{3}\right)$ & $1540 \mathrm{~m} / \mathrm{s}$ \\
Glass density $\left(\rho_{1}\right)$ & $2500 \mathrm{~kg} / \mathrm{m}^{3}$ \\
Fluid density $\left(\rho_{3}\right)$ & $1000 \mathrm{~kg} / \mathrm{m}^{3}$ \\
Attenuation coefficient $(\alpha)$ & $8.05 \mathrm{~Np} / \mathrm{m}-\mathrm{MHz}$
\end{tabular}

of first and second kinds, respectively, $\rho_{1}$ and $\rho_{3}$ are the density of the scatterer and surrounding fluid, respectively, $k_{1}, k_{2}$, and $k_{3}$ are the wavenumbers corresponding to compressional wave in scatterer, shear wave in scatterer, and sound wave in the surrounding medium, respectively, where $k=\frac{2 \pi f}{c}-i \alpha$ and $c$ is the velocity and $f$ is the frequency, $\alpha$ is the attenuation coefficient, $a$ is the scatterer diameter, and $r$ is the far-field distance from the center of the scatterer. The physical parameters are listed in Table 1.

From Eq. 6, the pressure of the scattered ultrasound wave was calculated in the forward direction at different scattering conditions. The scatterer diameter was 10,40 , and $70 \mu \mathrm{m}$, and the propagation path distance was $1000 \mu \mathrm{m}$. The propagation properties were similar to soft tissue, and the wave velocity was $1540 \mathrm{~m} / \mathrm{s}$. Pressure amplitude was measured along 18 to $41 \mathrm{MHz}$ frequency range for each scatterer size to achieve the pressure amplitude spectrum which is shown in Fig. 1. The total number of peaks and valleys (peak density) in each spectrum was calculated using a peak counting algorithm in MATLAB shown in Fig. 2 [24]. For 10, 40, and 70 $\mu \mathrm{m}$ spheres, the calculated peak density was 0,4 , and 21. From Fig. 1, it was observed that with the increase in scatterer size the pressure amplitude of the spectrum had been increased. Also, the variability in the spectrum amplitude was increased with the scatterer diameter which indicates the increase in peak density. Therefore, peak density can be defined as a parameter to express the level of scattering.

$\tan \xi(k a, v)=\left[-\frac{\left(k_{2} a\right)^{2}}{2} \frac{\frac{\left(k_{1} a\right) j_{1}^{\prime}\left(k_{1} a\right)}{\left(k_{1} a\right) j_{l}^{\prime}\left(k_{1} a\right)-j_{l}\left(k_{1} a\right)}-\frac{2\left(I^{2}+l\right) j_{1}\left(k_{2} a\right)}{\left(I^{2}+l-2\right) j_{1}\left(k_{2} a\right)+x_{2}^{2} j_{l}^{\prime \prime}\left(k_{2} a\right)}}{\frac{\left(k_{1} a\right)^{2}\left[\left(\frac{v}{1-2 v}\right) j_{1}\left(k_{1} a\right)-j_{1}^{\prime \prime}\left(k_{1} a\right)\right]}{\left(k_{1} a\right) j_{j}^{\prime}\left(k_{1} a\right)-j_{l}\left(k_{1} a\right)}-\frac{2\left(I^{2}+l\right)\left[j_{1}\left(k_{2} a\right)-x_{2} j_{1}^{\prime}\left(k_{2} a\right)\right]}{\left(I^{2}+l-2\right) j_{1}\left(k_{2} a\right)+\left(k_{2} a\right)^{2}}}\right]$

$\alpha_{l}, \beta_{l}, \delta_{l}$, and $\xi$ [23] are the scattering phase angle, $\Phi_{l}$ is the boundary impedance scattering phase angle, $v$ is the Poisson's ratio, $n_{l}$ is the spherical Bessel function
To understand the effect of ultrasound propagation path distance on peak density, the pressure spectrum was achieved from a $70 \mu \mathrm{m}$ scatterer for a distance of 1000 and 


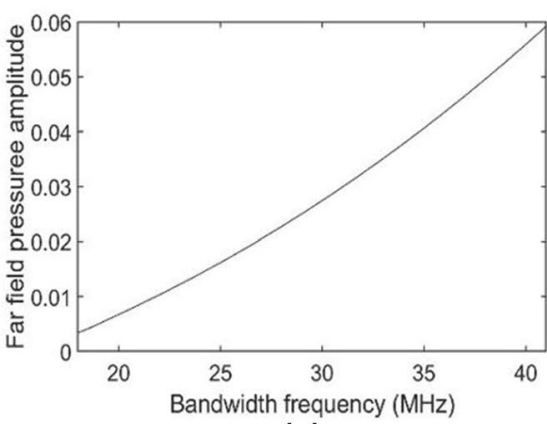

(a)

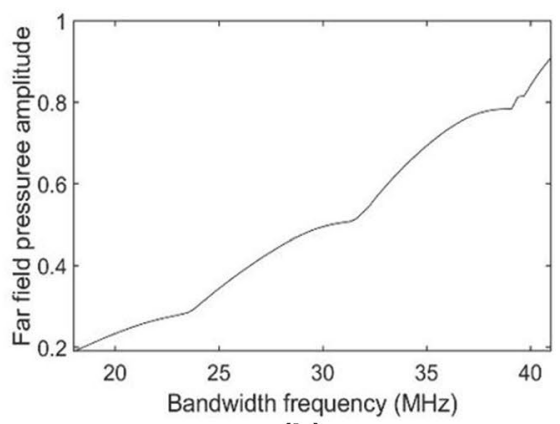

(b)

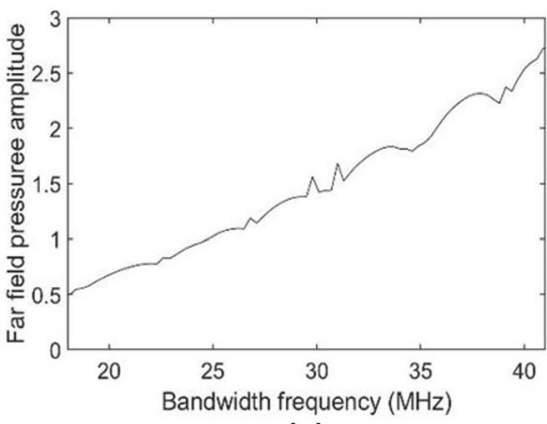

(c)

Fig. 1 Frequency spectrum for scatterer size of a $10 \mu \mathrm{m}, \mathbf{b} 40 \mu \mathrm{m}$, and c $70 \mu \mathrm{m} 4$

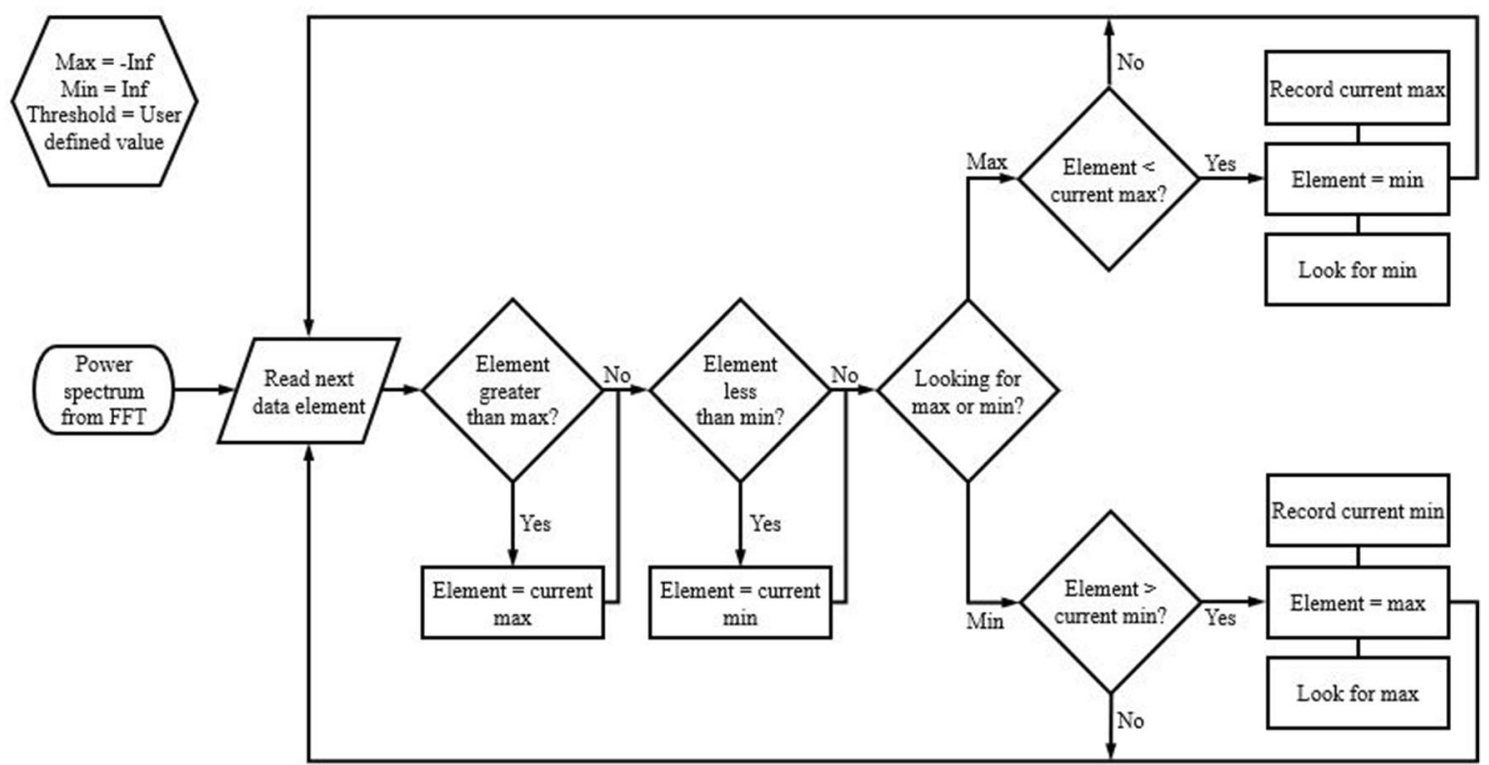

Fig. 2 Peak and valley counting algorithm

Fig. 3 Frequency spectrum for a scatterer of $70 \mu \mathrm{m}$ diameter at far field of $\mathbf{a} 500 \mu \mathrm{m}$ and $\mathbf{b}$ $1500 \mu \mathrm{m}$

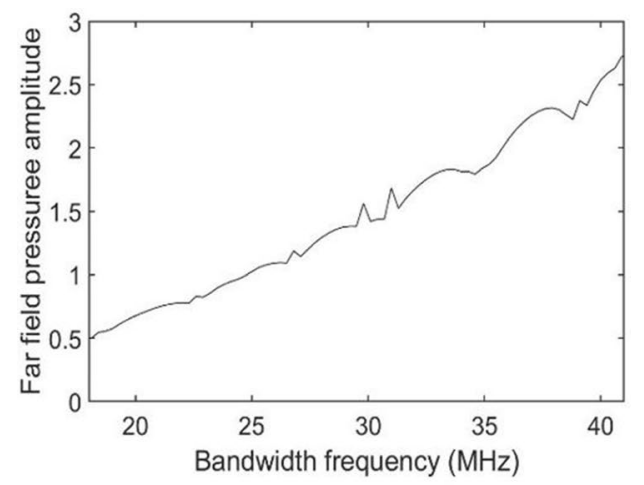

(a)

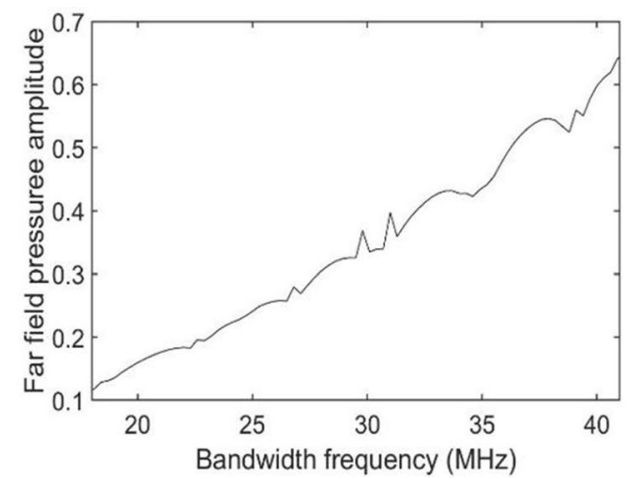

(b)
$3000 \mu \mathrm{m}$ and is plotted in Fig. 3. The far-field distance was measured from the center of the sphere which was half of the total travel path 500 and $1500 \mu \mathrm{m}$. From Fig. 3, it was observed that with increasing distance, only pressure amplitude level changed, but the pattern of the pressure amplitude remained the same. Since the wave was going 
through single scattering, the scattered wave was not getting interfered with any other wave in the way. Therefore, increasing travel distance only decreased the amplitude level because of attenuation, but the pattern, as well as the peak density, remained the same.

To understand the effect of multiple scattering created from several scatterers, computation model had been developed and evaluated.

\section{Computational analysis}

COMSOL Multiphysics 5.5a was used for the finite element simulation. The software was selected to solve partial differential equations in acoustics and solid mechanics. COMSOL solved the Helmholtz equation of acoustics in the fluid region of the model shown in Eq. 13

$\nabla \cdot\left(-\frac{1}{\rho_{c}}\left(\nabla p_{t}-q_{d}\right)\right)-\frac{k_{\text {eq }}^{2}}{\rho_{c}} p_{t}=Q_{m}$

$k_{\text {eq }}$ is the equivalent wave number defined for wave velocity $c_{c}$ in fluid and frequency $f$ shown as

$k_{\text {eq }}^{2}=\left(\frac{2 \pi f}{c_{c}}-i \ln (10) \frac{\alpha}{20}\right)^{2}-k_{z}^{2}=k^{2}-k_{z}^{2}$

Here, $\alpha$ is the attenuation coefficient of the medium. In this model, the out-of-plane wave number $k_{z}$, monopole and dipole source term $Q_{m}$ and $q_{d}$ were set to zero. $\rho_{c}$ is the fluid density. The total pressure $p_{t}$ is the summation of the background pressure field $\left(p_{b}\right)$ and backscatter pressure field $\left(p_{s}\right)$. In the model, the background pressure field was expressed as wave propagation in $\hat{e_{x}}$ direction defined as $p_{b}=p_{0} e^{-k x}$. After applying all the adjustments, the modified Helmholtz equation became

$\nabla^{2} p_{t}+k^{2} p_{t}=0$

In the solid region, the scatterers were modeled as linearly elastic isotropic material for which 2D Navier's equation was solved

$-(2 \pi f)^{2} \rho_{s} u=\nabla \cdot S+\mathrm{Fe}^{i \phi}$

In this equation, $f$ is the sound frequency, $S$ is the Cauchy stress tensor, $\rho_{s}$ is the solid density, $\phi$ is the phase component of the force, $F$ is the acting force on solid, and $u$ denotes the solid displacement field.

The equations in the acoustics and solid mechanics were coupled by the following boundary conditions

$n \cdot \frac{1}{\rho_{c}} \nabla p_{t}=-n \cdot u_{\mathrm{tt}}$
$F_{A}=p_{t} n$

$n$ is the normal unit vector to the boundary, $u_{\mathrm{tt}}$ is the structural acceleration, and $F_{A}$ is the acting load on solid.

\subsection{Model description}

The computational model consisted of a solid and fluid region. A perfectly matched layer was introduced to perform as a non-reflecting boundary layer to have an uninterrupted wave propagation through the boundary where the wave pressure was recorded. To select the proper mesh size, mesh size was decreased gradually, and for each mesh size, peak density was measured. For the mesh size of one-eighth of the wavelength, the peak density result was converged for all the models. Therefore, it was selected as the maximum mesh size for all models. To capture perfect multiple scattering, the scatterers were distributed randomly. The medium layer area was $1 \times 1 \mathrm{~mm}^{2}$ where the sound wave would travel $1 \mathrm{~mm}$ distance. To get the effect of sample thickness or various travel distances, two more models having $1 \times 2$ and $1 \times 3$ $\mathrm{mm}^{2}$ layer area were generated. For each of these three models, the number of scatterers used was 1, 5, and 9 per unit $(\mathrm{mm})$ wave travel distance. Also, the scatterer size was selected as 10,40 , and $70 \mu \mathrm{m}$. Therefore, a total of 27 models were generated.

To mimic a soft tissue medium, the fluid region's density $\left(\rho_{c}\right)$ was kept $1000 \mathrm{~kg} / \mathrm{m}^{3}$, sound velocity $\left(c_{c}\right)$ was considered as $1540 \mathrm{~m} / \mathrm{s}$, and the attenuation coefficient $(\alpha)$ was selected as $70 \mathrm{~dB} / \mathrm{m}$ at $1 \mathrm{MHz}$ [25]. Solid structures density $\left(\rho_{s}\right)$ was $2500 \mathrm{~kg} / \mathrm{m}^{3}$, Poisson's ratio $(v)$ was 0.22 , Young's modulus $\left(E_{Y}\right)$ was $68 \mathrm{GPa}$, and sound speed $\left(c_{s}\right)$ was $5570 \mathrm{~m} / \mathrm{s}$. Figure 4 shows a typical computational model geometry used in this analysis having 5 scatterers of $40 \mu \mathrm{m}$ diameter.

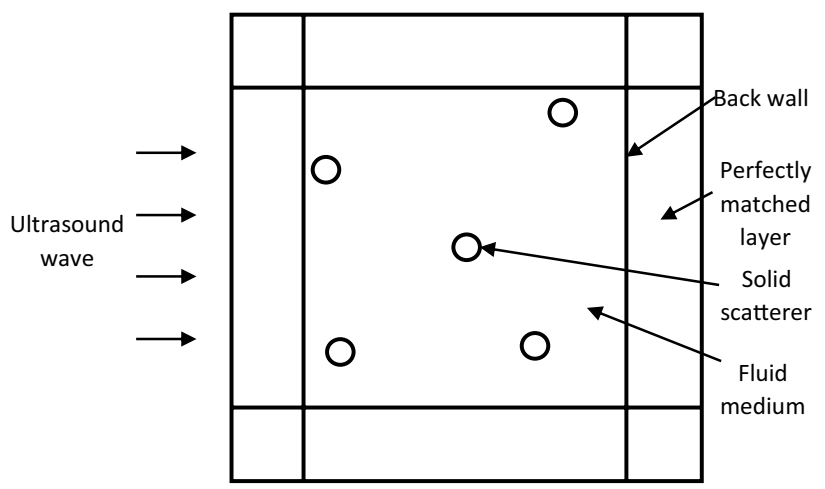

Fig. 4 COMSOL model geometry for ultrasound analysis 


\subsection{Simulation parameters}

To achieve the frequency spectrum, the frequency was increased by $100 \mathrm{kHz}$ starting from 18 to $41 \mathrm{MHz}$. For each step frequency, the model calculated scattered pressure. Since the objective was to evaluate pitch-catch wave propagation, the pressure of the forward scattered wave was calculated on the far wall shown in Fig. 4. After evaluating the average acoustic pressure on the back wall for each frequency step, it was plotted with respect to the frequency to achieve the frequency spectrum. In the end, MATLAB was used to evaluate the data for measuring peak density. This procedure was repeated for all the 27 models to compute the peak density.

\subsection{Results}

Figure 5 depicts the peak density for all the models calculated at each thickness level with varying scatterer size and number. An increasing trend of peak density was observed with the increase in each parameter. From the analytical study, it was understood that with increasing scatterer size the peak density increased from the single scattering. But when it came to multiple scattering, all the parameters which were thickness, scatterer size, and number contributed toward the multiple scattering to change the peak density. This result makes peak density a very sensitive parameter while characterizing a material microstructure from every aspect.

Since peak density increased with increasing thickness, the effect of ultrasound attenuation on multiple scattering needed to be explored. Therefore, two sets of models were simulated. The first set of 9 models consists of 1, 2, and $3 \mathrm{~mm}$ propagation distance with each having a total number of scatterers 1,5 , and 9 , respectively. The scatterer diameter was kept as $40 \mu \mathrm{m}$. The second set of 9 models had 1,2, and $3 \mathrm{~mm}$ propagation distance with each having scatterer diameter 10,40 , and $70 \mu \mathrm{m}$, respectively. The total number of scatterers was 5 in the second set of models.

Figure 6 shows the effect of ultrasound attenuation in multiple scattering as well as peak density. Figure 6 a shows that peak density decreases with increasing thickness for the same number of scatterer with a constant diameter. This happened due to amplitude attenuation at high frequency for the same level of acoustic scattering. But with the increasing number of scatterers, peak density started to increase in every thickness level. Similar results were found in the case of scatterer size in Fig. $6 \mathrm{~b}$ where peak density was decreasing with increasing thickness. But it started to increase in each thickness level with increasing scatterer size. This explains that by increasing scatterer number or size, the multiple scattering level increases which results in higher peak density for the same level of attenuation. This validates the increasing trend of peak density along with increasing thickness in Fig. 5. Because with increasing thickness, the total scatterer number was also increasing since the scatterer number was selected on a "per unit thickness" basis. This increasing amount of scatterer overcomes the effect of attenuation and raised the peak density value. But since ultrasound eventually attenuates faster at high frequency, it limits this peak density trend to a certain thickness level.

With the random distribution of scatterers, an important associated factor came into consideration which was the distance between the scatterers. Since the scatterers were distributed randomly in the medium, the distance among them was not controlled intentionally to support the randomness. But after understanding the sensitivity level of peak density, it became an essential

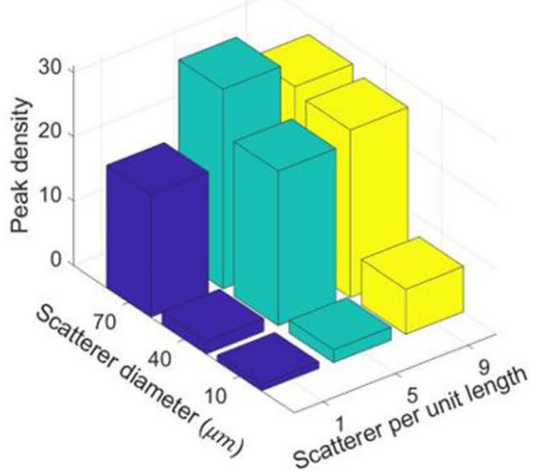

(a)

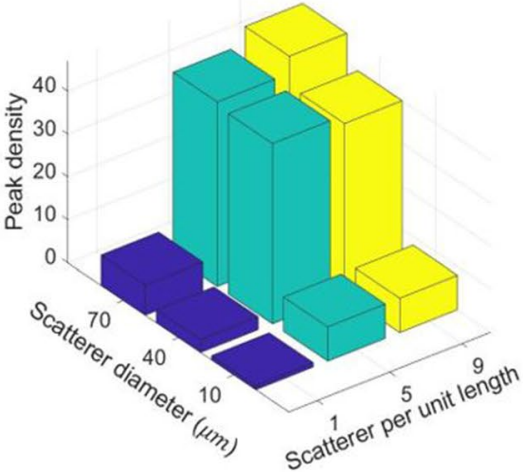

(b)

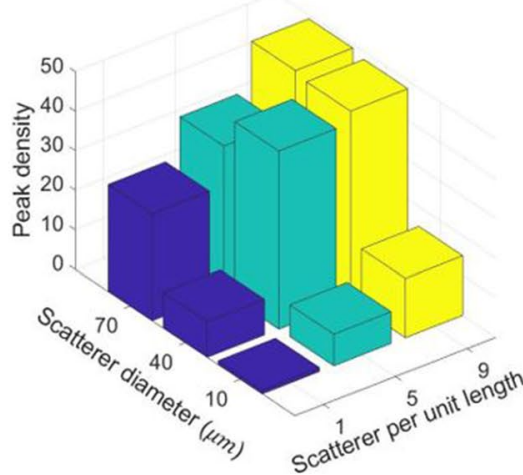

(c)

Fig. 5 Computational peak density value for different scatterer numbers and diameters at wave propagation distance of a $1 \mathrm{~mm}$, $\mathbf{b} 2 \mathrm{~mm}$, and $\mathrm{c} 3 \mathrm{~mm}$ 
Fig. 6 Peak density at increasing thickness with a increasing number of scatterer, $\mathbf{b}$ increasing scatterer size

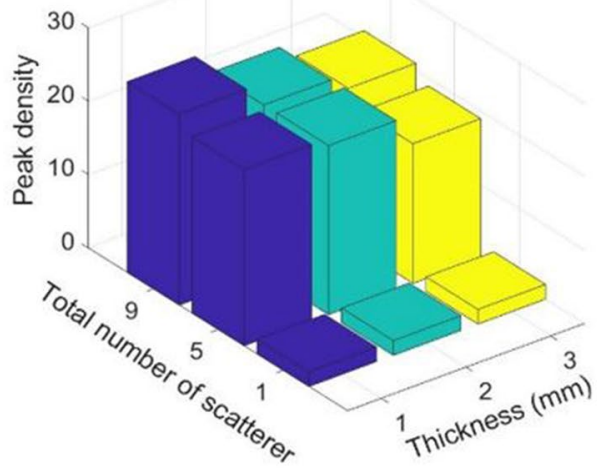

(a)

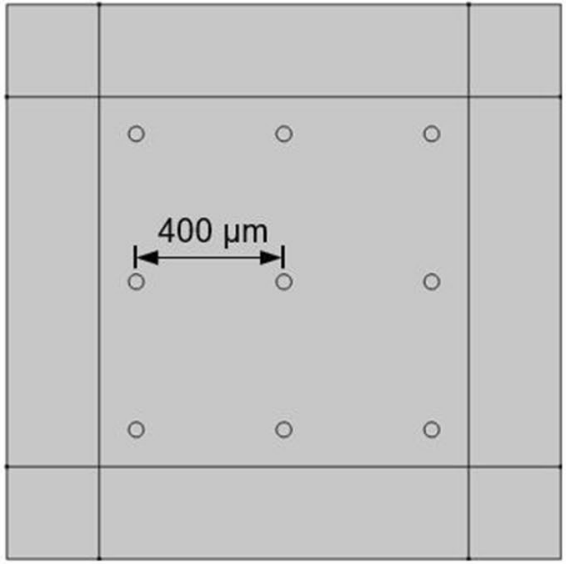

(a)

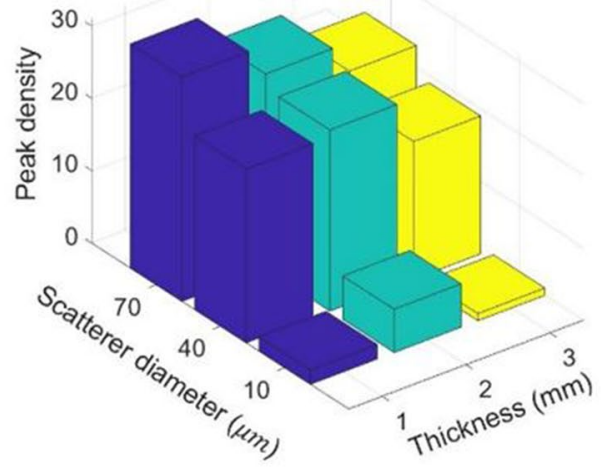

(b)

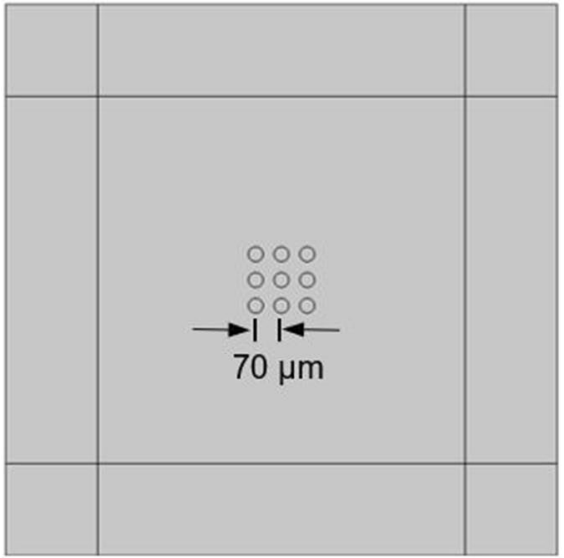

(b) concern to evaluate the inter-scatterer distance effect on peak density while keeping every other parameter constant. A simple computational model of $1 \times 1 \mathrm{~mm}^{2}$ layer area was developed to explore this phenomenon; 9 glass scatterers were placed in a rectangular array inside the fluid region. The scatterer diameter was selected to be $40 \mu \mathrm{m}$. The scatterers were placed in equal distance from each other; 7 models were developed by varying the distance between the scatterers which were 400, 300, 250, 150,100 , and $70 \mu \mathrm{m}$. Figure 7 shows two of the models having the inter-scatterer distance of 400 and $70 \mu \mathrm{m}$.

Figure 8 shows the peak density plotted against the inter-scatterer distance. It was evident that the distance between the scatterers also played a vital role in the peak density variation. Especially when the distance increased, the scattered wave interfered with each other along the whole way while increasing the amount of scattering. It resulted in higher peak density. This phenomenon gave the peak density an advantage to detect the scatterer orientation during the analysis.

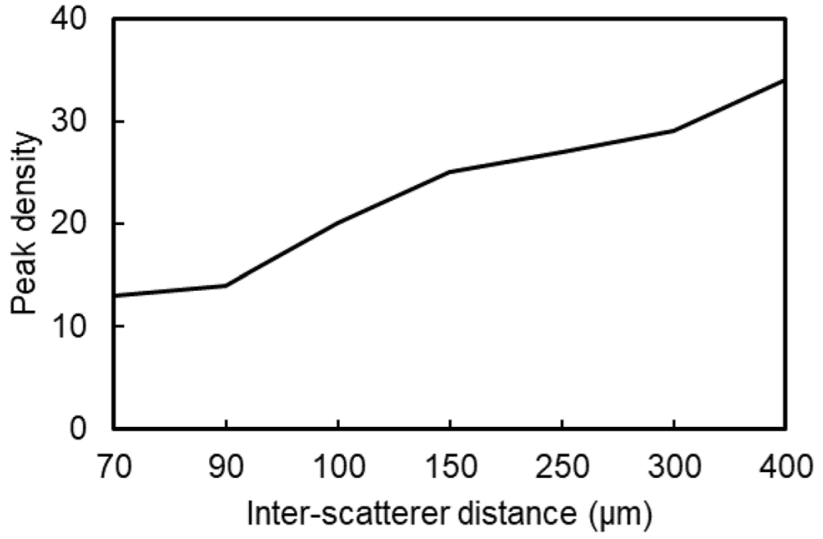

Fig. 8 Peak density vs inter-scatterer distance

\section{Experimental analysis}

An experimental setup was built to perform ultrasound analysis to evaluate the peak density. 


\subsection{Sample preparation}

The agarose-based phantom was prepared from highpurity agarose, water, n-propanol, evaporated milk, and thimerosal [26]. First, a mixture of agarose, water, and $\mathrm{n}$-propanol was made and heated to $90^{\circ} \mathrm{C}$. The weight ratio of those materials was 23:1:2, respectively. Then, another mixture of evaporated milk and thimerosal was made and heated in $68^{\circ} \mathrm{C}$. The weight ratio was 1.33:800, respectively. After that, both mixtures were added together in $55^{\circ} \mathrm{C}$ and then solidified. To introduce microstructural variation as well as to enhance the acoustic scattering properties of the soft material in terms of acoustic scattering, glass microbeads were inserted inside the sample. To keep similarity with the computational model, three types of glass microbeads of diameter 8-12 $\mu \mathrm{m}, 35-45 \mu \mathrm{m}$, and 65-75 $\mu \mathrm{m}$ were inserted inside the phantom. Each type of microbeads was mixed with the phantom in three different volumetric ratios- $1 \%, 5 \%$, and $9 \%\left(V_{\text {microbead }} / V_{\text {total }}\right)$. Also, three levels of sample thickness were selected which were $1 \mathrm{~mm}, 2 \mathrm{~mm}$, and $3 \mathrm{~mm}$. Therefore, there were 27

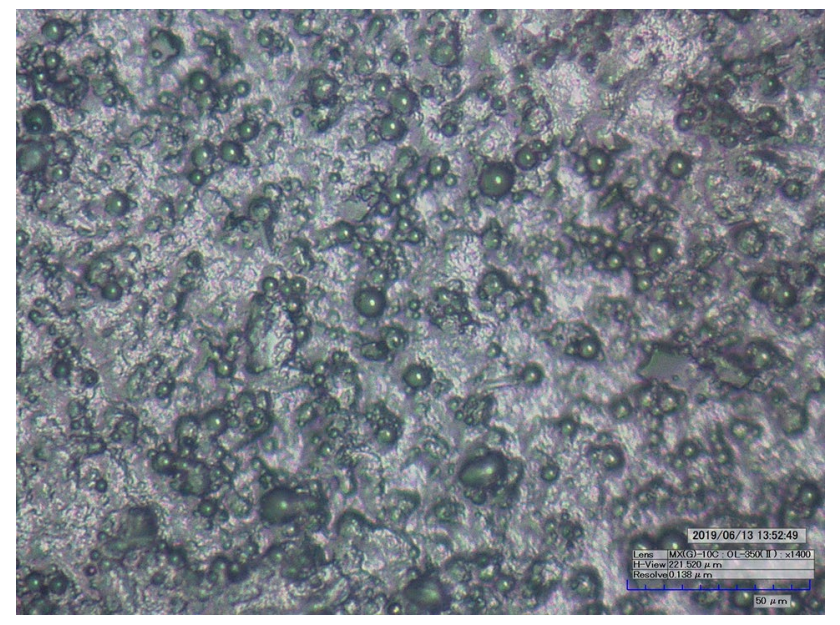

Fig. 9 Agarose-based phantom with glass microbeads types of phantom generated for the experimental analysis. The image of one of the phantoms with glass scatterers is shown in Fig. 9. The image was taken through an optical microscope and a digital camera.

\subsection{Experimental setup}

Figure 10 shows the experimental setup which consisted of two ultrasound transducers $(25 \mathrm{MHz}, 2 \mathrm{~mm}$ diameter, TransducerWorks, Centre Hall, PA, USA), a pulser receiver system (UTEX UT 340, UTEX Scientific Instruments Inc. Mississauga, Ontario L5L 1A3, Canada) and a mix-domain oscilloscope (Tektronix MDO 3052, Tektronix, Inc. Beaverton, OR 97,077, USA), coupling gel (Aquasonic 100, Walmart INC), height-adjustable station for phantom and transducer, and tissue-mimicking phantom. The coupling gel was used to save the transducers from the damage occurring from the impedance mismatch between the transducer crystal and phantom. Also, the gel ensured less amount of intensity loss of the high-frequency wave along its way from the transducer to the phantom. For the ultrasonic transducers, the $-6 \mathrm{~dB}$ frequency bandwidth was in the range of $18-41 \mathrm{MHz}$. The station for transducers and phantom was made in a way that either phantom or any transducer position could be adjusted by changing the height of the structure. This was done to adjust the setup with different thicknesses of phantoms.

For each of the phantoms, the ultrasound analysis was conducted in 14 different places to calculate the peak density. Final peak density was then determined from the average of 14 results from each sample.

\subsection{Data acquisition}

To proceed with the analysis, first, the high-frequency ultrasound signal was sent from the pulse receiver. Table 2 lists all the pulse parameters selected for signal generation. Figure 11 shows the experimental process steps. A pitch-catch technique was used to conduct the ultrasound
Fig. 10 Photograph of ultrasound analysis setup

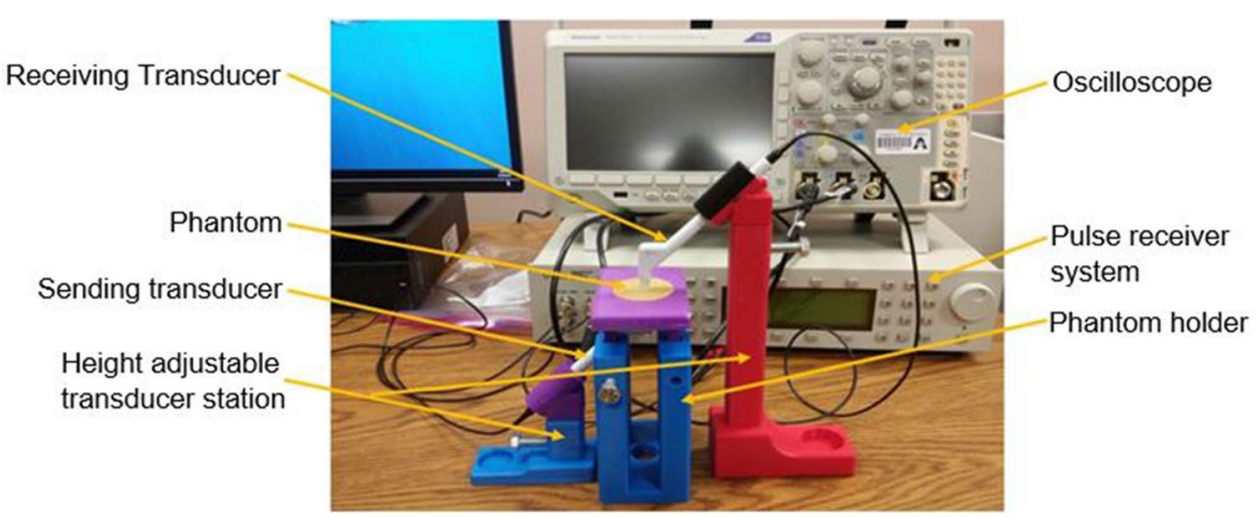


Table 2 Ultrasound parameter

\begin{tabular}{ll}
\hline Parameter & Value \\
\hline Voltage & $100 \mathrm{~V}$ \\
P/C gain & $48 \mathrm{~dB}$ \\
Repetition rate & $19 \mathrm{kHz}$ \\
Pulse width & $20 \mathrm{~ns}$ \\
\hline
\end{tabular}

analysis. Both transducers were aligned on top of each other to ensure proper sound propagation. The ultrasound signal was sent from one transducer which went through the coupling gel, phantom, and received by another transducer. The received signal was again sent to the pulse receiver for further amplification. After that, the signal was stored in the computer through the oscilloscope for further signal processing and peak density calculation.

\subsection{Signal processing and peak density evaluation}

The time-domain signal was stored in the computer from the oscilloscope and the signal processing was performed through MATLAB. The first step of the signal processing was windowing the signal. Stromer et al. showed that the rectangular window performed better compared to Hamming, Hann, Tukey, and Blackman windowing function while calculating the peak density [19]. Therefore, a rectangular window was applied on the time domain signal to capture only the pulse generated by the transducer crystal. The windowed signal was then transformed from the time domain to the frequency domain through Fast Fourier Transformation (FFT). To eliminate any possible systemic consequences from pulse receiver and transducer, the signal was normalized by a reference signal. To generate a reference signal, first, the ultrasound was generated in the same pitch-catch setup and guided through the coupling gel only. The signal then went through all the signal processing steps stated above. Then, the FFT sample signals pressure amplitude was divided by the pressure amplitude of the reference signal on each frequency step of the frequency bandwidth to generate the calibrated signal. In the end, the number of peaks was counted from the calibrated signal between the 18 and $41 \mathrm{MHz}$ frequency range. This procedure was repeated for each of the 27 types of samples for 14 times. Figure 12 shows a typical numerical frequency spectrum (thickness $3 \mathrm{~mm}$ and scatterer diameter $70 \mu \mathrm{m}$ with 9 scatterers per unit length) and an experimental frequency spectrum (thickness $3 \mathrm{~mm}$, scatterer diameter 65-75 $\mu \mathrm{m}$, and volume fraction $9 \%$ ).

\subsection{Results}

Figure 13 shows the peak density calculated from the experimental analysis at each thickness level. Since there were 14 repetitive measurements conducted for each sample combination, the error bar is provided in Fig. 13. The maximum error was \pm 1.65 which indicates that the peak density difference between various sample combinations is significant. The peak density result was similar to the computational values. The experimental values also indicated that the peak density is sensitive toward all the parameters in the model. The highest value of peak density was received from the sample having the maximum thickness, scatterer size, and quantity.

Since three different parameters were affecting the multiple scattering as well as the peak density value, it was important to statistically analyze the data to find out the significance level of each parameter. To achieve this, a full factorial DOF analysis was conducted consisting of 3 factors having three levels each to calculate the main effect of the parameters as well as interaction effects. The results are shown in Fig. 14.

From the analysis, it was found that peak density is most sensitive toward volumetric ratio, then scatterer diameter, and lastly toward sample thickness. Although the scatterer size was only responsible for the peak density variation in
Fig. 11 Schematic diagram of the ultrasound analysis process in pitch-catch setup

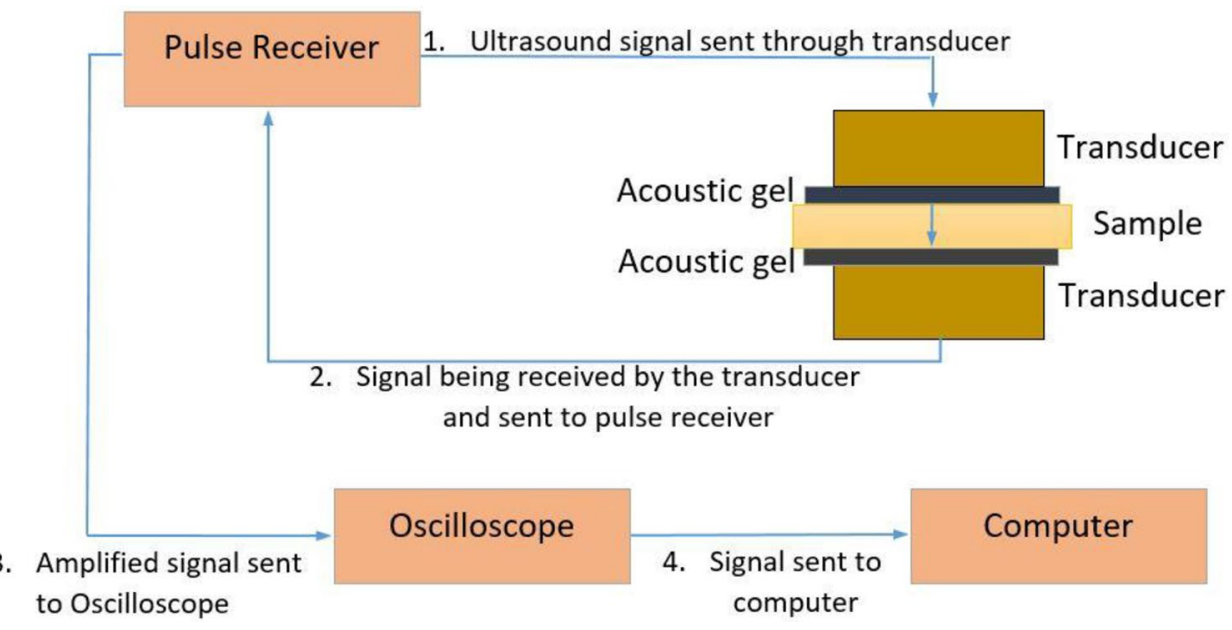

SN Applied Sciences A SPRINGER NATURE journa 
Fig. 12 Frequency spectrum from a computation model and $\mathbf{b}$ experimental model

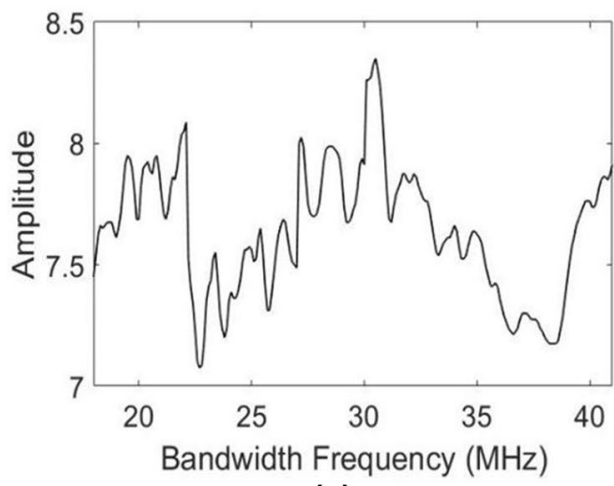

(a)
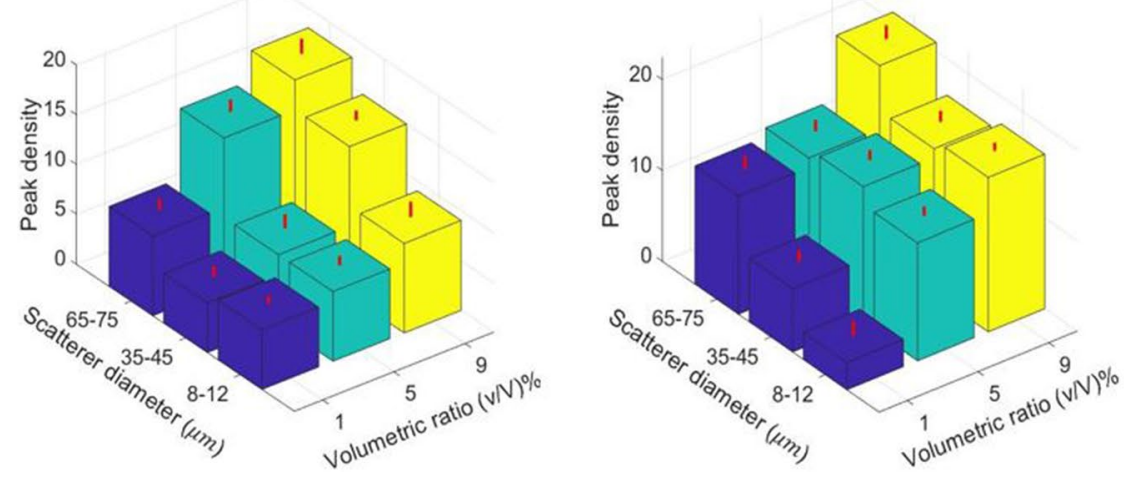

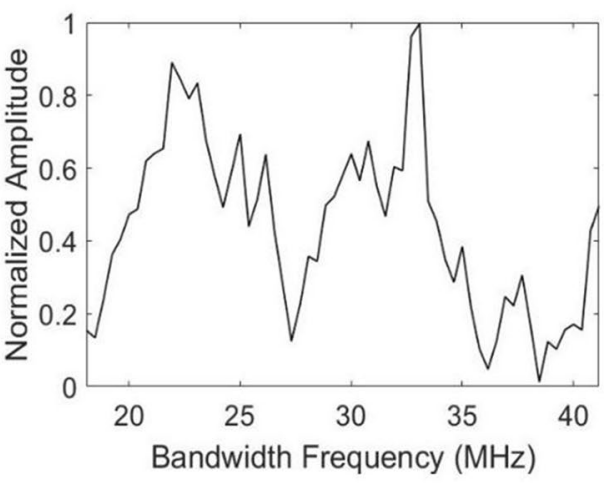

(b)

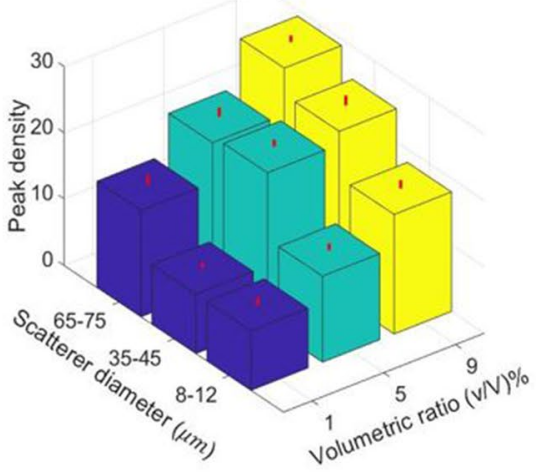

Fig. 13 Experimental peak density value for different scatterer numbers and diameters at wave propagation distance of a $1 \mathrm{~mm}$, $\mathbf{b} 2 \mathrm{~mm}$, and $\mathrm{c} 3 \mathrm{~mm}$

single scattering, the number of scatterers also became effective toward the variation, in case of multiple scattering. Also, the thickness or the wave travel distance became active toward the change of peak density in case of multiple scattering. Furthermore, there was no parameter interaction significance found in the analysis. It concludes that if one of the three parameters were kept constant, it would not affect the other parameters contribution toward the peak density variation.

\section{Discussion}

In this study, the sound scattering of high-frequency ultrasound in a pitch-catch setup was evaluated through analytical, computational, and experimental analysis. The main goal was to establish peak density as a sensitive parameter of high-frequency ultrasound. To achieve the goal, peak density was established as a function of sound scattering. From the analytical study, while assessing the single scattering, peak density was found to be very sensitive toward the change of scatterer size. Also, it showed an increasing trend with the increase in scatterer size. But in case of changing the propagation path, the peak density did not change since scattered wave did not have any interaction with other waves on their extended travel path. Therefore, to explore the effect of travel path on peak density, it was necessary to explore multiple scattering where the scattered wave would continuously interact with other scattered waves from different scatterers through the propagation path.

In the computational modeling of multiple scattering, along with scatterer size and propagation path, the number of scatterers was also considered as another factor for peak density. Through computation modeling, it was observed that with increasing scatterer size, propagation distance, and scatterer number, the peak density increases. The effect of attenuation on peak density becomes less significant with scatterers being added per unit length at an increasing level of thickness. This gave peak density an advantage while using high-frequency ultrasound before the signal fully attenuates. Furthermore, the effect of the randomness of the scatterer position was also evaluated through a simplified model. The factor which was evaluated in that analysis was the inter-scatterer distance. It was observed that with increasing inter-scatterer distance the 


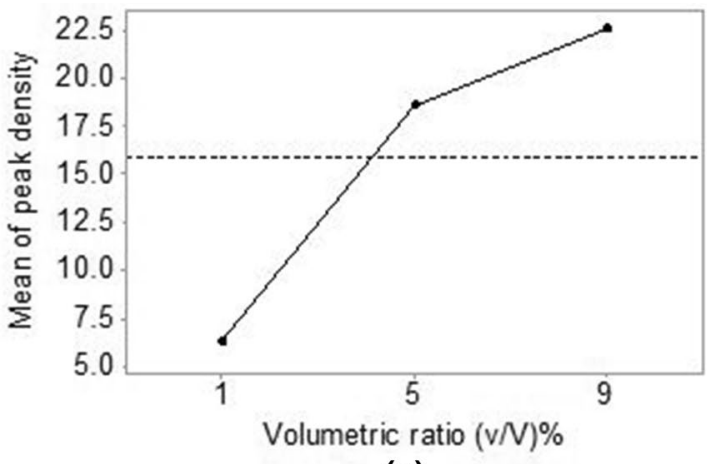

(a)

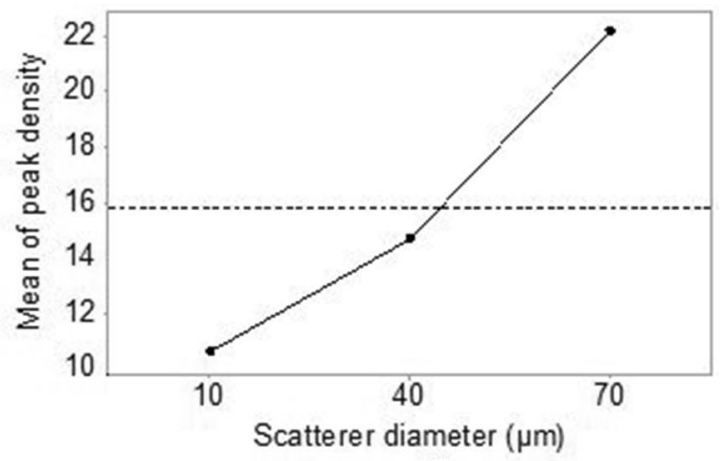

(b)

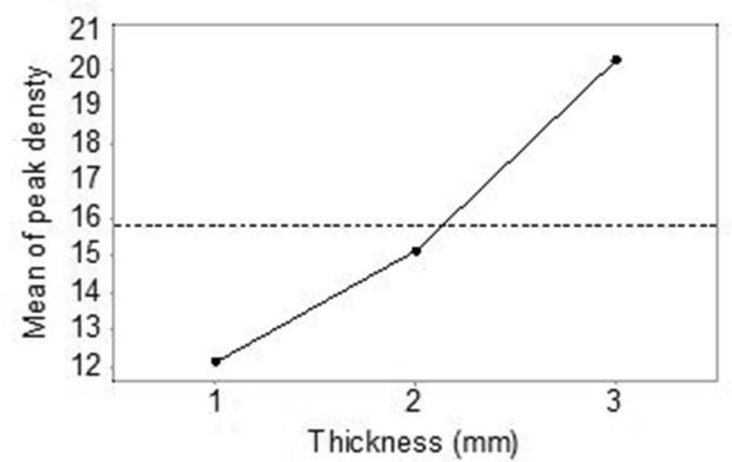

(c)

Fig. 14 Statistical significance of a volumetric ratio, $\mathbf{b}$ scatterer diameter, and $\mathbf{c}$ sample thickness on peak density

peak density also increases. As the inter-scatterer distance decreases, the scattering wave interference gets limited in that smaller region of scatterers and does not sustain further while propagating as there are no other waves to interact with.

The experimental results validated the computational findings by showing similar peak density trends in terms of changing scatterer number, size, and propagation distance. It was important to observe the level of influence for each of the factors. Therefore, a full factorial design including three different levels of scatterer number, size, and propagation distance was created considering peak density the outcome. From the statistical analysis, it was observed that the number of scatterers affects the peak density the most compared with the other two factors. The increasing number of scatterers increases the level of multiple scattering since more scattered waves from multiple scatterers get to interact with each other. Therefore, peak density can be shown as a function of acoustic scattering. As per the experimental setup, peak density can be used toward the detection of $\mathrm{mm}$ scale soft material. It has been studied that the inclusion of glass microbeads in tissue phantom increases the scattering quality of the phantom [26]. Furthermore, controlled dispersion of glass microspheres was used to mimic abnormal mass in soft tissues like calcified fibroadenoma containing stromal and epithelial cells [27]. Since a higher amount of glass beads can provide a higher peak density value compared to fewer beads, this parameter can be used to evaluate tissue abnormality by utilizing acoustic scattering. The authors did not find any previous research on phantom preparation by glass microbeads for mimicking either lobular or ductile carcinoma. But with the results obtained in this study, it can be concluded that peak density can be a very promising parameter for ultrasound analysis in cancer detection.

\section{Conclusion}

Ultrasound scattering was evaluated in the study through the pitch-catch technique in high-frequency level. The scattering was evaluated with respect to various scatterer sizes, numbers, and wave propagation distances. To quantify the scattering, the peak density of the frequency spectrum of the scattered wave was evaluated. It was found that the variation in scatterer number in the medium's microstructure affects sound scattering the most which resulted in a huge impact on the peak density diversification. Other than that, scatterer size and wave propagation distance also play important roles in the peak density variation. This makes peak density a very sensitive parameter 
to the microstructural change. Also, the inter-scatterer distance plays an important role in the multiple scattering for providing peak variation in case of having the same number of scatterer in a sample in different orientations. Therefore, while working with the pitch-catch technique, peak density can be used to measure the level of multiple scattering while evaluating detailed microstructural characteristics.

\section{Compliance with ethical standards}

Conflict of interest On behalf of all authors, the corresponding author states that there is no conflict of interest.

\section{References}

1. Pavlin CJ, Harasiewicz K, Sherar MD, Foster FS (1991) Clinical use of ultrasound biomicroscopy. Ophthalmology 98:287-295. https ://doi.org/10.1016/S0161-6420(91)32298-X

2. Reinstein DZ, Srivannaboon S, Gobbe M, Archer TJ, Silverman $\mathrm{RH}$, Sutton H, Coleman DJ (2009) Epithelial thickness profile changes induced by myopic LASIK as measured by Artemis very high-frequency digital ultrasound. J Refract Surg 25:444-450. https://doi.org/10.9999/1081597X-20090422-07

3. Passmann C, Ermert H (1996) A 100-MHz ultrasound imaging system for dermatologic and ophthalmologic diagnostics. IEEE Trans Ultrason, Ferroelectr, Freq Control 43(4):545-552

4. Turnbull DH, Starkoski BG, Harasiewicz KA, Semple JL, From L, Gupta AK, Sauder DN, Foster FS (1995) A 40-100 MHz B-scan ultrasound backscatter microscope for skin imaging. Ultrasound Med Biol 21:79-88

5. (2019) What Is Breast Cancer? | Breastcancer.org, https://www. breastcancer.org/symptoms/understand_bc/what_is_bc

6. Dagogo-Jack I, Shaw AT (2018) Tumour heterogeneity and resistance to cancer therapies. Nat Rev Clin Oncol 15(2):81

7. Pleijhuis RG, Graafland M, De Vries J, Bart J, De Jong JS, Van Dam GM (2009) Obtaining adequate surgical margins in breast-conserving therapy for patients with early-stage breast cancer: current modalities and future directions. Ann Surg Oncol 16:27172730. https://doi.org/10.1245/s10434-009-0609-z

8. Jacobs $L$ (2008) Positive margins: the challenge continues for breast surgeons. Ann Surg Oncol 15:1271-1272. https://doi. org/10.1245/s10434-007-9766-0

9. Singletary SE (2002) Surgical margins in patients with earlystage breast cancer treated with breast conservation therapy. Am J Surg 184:383-393. https://doi.org/10.1016/S0002 -9610(02)01012-7

10. Russo AL, Arvold ND, Niemierko A, Wong N, Wong JS, Bellon JR, Punglia RS, Golshan M, Troyan SL, Brock JE, Harris JR (2013) Margin status and the risk of local recurrence in patients with earlystage breast cancer treated with breast-conserving therapy. Breast Cancer Res Treat 140:353-361. https://doi.org/10.1007/ s10549-013-2627-6

11. Doyle $T E$, Factor $R E$, Ellefson $C L$, Sorensen $K M$, Ambrose $B J$, Goodrich JB, Hart VP, Jensen SC, Patel H, Neumayer LA (2011) High-frequency ultrasound for intraoperative margin assessments in breast conservation surgery: A feasibility study. BMC Cancer 11:444. https://doi.org/10.1186/1471-2407-11-444
12. Langton CM, Njeh CF (eds) (2016) Quantitative ultrasound. In: The physical measurement of bone. CRC Press, Boca Raton, pp 412-474

13. Lizzi FL, Greenebaum M, Feleppa EJ, Elbaum M, Coleman DJ (1983) Theoretical framework for spectrum analysis in ultrasonic tissue characterization. J Acoust Soc Am 73:1366-1373. https:// doi.org/10.1121/1.389241

14. Lizzi FL, Ostromogilsky M, Feleppa EJ, Rorke MC, Yaremko MM (1987) Relationship of Ultrasonic Spectral Parameters to Features of Tissue Microstructure. IEEE Trans Ultrason Ferroelectr Freq Control 34:319-329. https://doi.org/10.1109/T-UFFC.1987.26950

15. Kremkau FW, Forsberg F (2016) Sonography : principles and instruments, 9 th ed. Elsevier, St. Louis, MO

16. Doyle TE, Cervantes M, Thompson LA, Roring JE, Grover MA, Chappell JA, Curtis BJ, Stiles JE, Borget BD (2013) Determining breast pathology in surgical margins with high-frequency ultrasound: phantom and numerical simulations. Proc Mtgs Acoust 19:075081. https://doi.org/10.1121/1.4800329

17. Dalton CE, Wagner GM, Al-Ghaib HA, Doyle TE (2018) Abstract P2-03-13: pathology differentiation during breast conservation surgery using high-frequency ultrasound and peak frequency distribution. Cancer Res. 78:P2-03-13

18. Butler AP, Omer RK, Doyle TE (2013) High-frequency ultrasound of breast tissue phantoms with histology mimicking microstructures. J Acoust Soc Am 134(5):4215-4215

19. Stromer J, Ladani L (2018) Examination of a spectral-based ultrasonic analysis method for materials characterization and evaluation. Biomed Signal Process Control 40:454-461. https:// doi.org/10.1016/j.bspc.2017.10.010

20. Stromer J, Ladani L (2018) Influence of microstructure on the high-frequency ultrasound measurement of peak density. J Nondestruct Eval Diagn Progn Eng Syst. https://doi. org/10.1115/1.4041067

21. Faran JJ (1951) Sound scattering by solid cylinders and spheres. J Acoust Soc Am 23:405-418. https://doi.org/10.1121/1.19067 80

22. Stanton TK (1990) Sound scattering by spherical and elongated shelled bodies. J Acoust Soc Am 88:1619-1633. https://doi. org/10.1121/1.400321

23. Hickling R (1962) Analysis of echoes from a solid elastic sphere in water. J Acoust Soc Am 34:1582-1592. https://doi. org/10.1121/1.1909055

24. Stromer J, Ladani L (2016) Investigating ultrasound imaging in the frequency domain for tissue characterisation. Nondestruct Test Eval 31:209-218. https://doi.org/10.1080/10589 759.2015.1093627

25. Goldstein A, Powis RL (1999) Medical ultrasonic diagnostics. Elsevier Masson, Paris

26. Madsen EL, Frank GR, Dong F (1998) Liquid or solid ultrasonically tissue-mimicking materials with very low scatter. Ultrasound Med Biol 24:535-542. https://doi.org/10.1016/j.procs .2016 .09 .052

27. Vieira SL, Pavan TZ, Junior JE, Carneiro AAO (2013) Paraffingel tissue-mimicking material for ultrasound-guided needle biopsy phantom. Ultrasound Med Biol 39:2477-2484. https:// doi.org/10.1016/j.ultrasmedbio.2013.06.008

Publisher's Note Springer Nature remains neutral with regard to jurisdictional claims in published maps and institutional affiliations. 